criticism. The latter two helped to overcome stalemates in the calculations.

\section{REFERENCES}

[1] B. Zotter and K. Bane, Stanford Linear Accelerator Center PEP-Note $308,1979$.

[2] J. Chandezon, G. Cornet, and G. Raoult, C. R. Acad. Sci. Paris, vol.
277, ser. B, p. 403, 1973.

[3] O. R. Asfar and A. H. Nayfeh, IEEE Trans. Microwave Theory Tech., vol. MTT-23, no. 9, p. 728, 1975.

[4] M. Chatard-Moulin and A. Papiernik, IEEE Trans. Nucl. Sci., vol. NS-26, no. 3, p. $3523,1979$.

[5] R. K. Cooper and P. L. Morton, Stanford Linear Accelerator Center Informal Rep., Apr. 1980

[6] E. Courant and H. Snyder, Annals Phys., vol. 3, no. 1, p. 1, 1958: also in M. Sands, Stanford Linear Accelerator Center SLAC-121, 1970.

\title{
Impedance Transformations for the Generalized Reflection Modulator
}

\author{
HARRY A. ATWATER, SENIOR MEMBER, IEEE
}

\begin{abstract}
A bstract-A procedure is given for obtaining the impedance transformer which will produce a prescribed pair of phasor reflection coefficients by transformation from a given pair of impedance states terminating the transformer. The transformer may be used in a general two-state reflection modulator: as a phase shifter with phase change at constant amplitude or as an amplitude modulator with level shift at constant or variable phase. The condition governing the reflection coefficients available from a given pair of impedance states is given. Examples and limitations are discussed.
\end{abstract}

\section{INTRODUCTION}

$\mathrm{T}$ HE REFLECTION modulator is useful as a phase shifter and as an amplitude modulator for digital modulation [1]-[8]. In typical applications, the modulator may be composed of a circulator or hybrid coupler terminated in a pair of variable semiconductor impedance elements. The transmission factor of the modulator is then proportional to the reflection coefficient of the terminations. The application of impedance transformation to modify the reflection coefficients of the terminating impedances was introduced by Kawakami [9] and by Kurokawa and Schlosser [10]. In the present paper, a unified treatment of the reflection phase shifter and reflection-type amplitude modulator is provided. A procedure is given for the calculation of the design parameters of a lossless impedance transformer to produce a specified pair of reflection coefficient values from a given pair of impedance

Manuscript received September 18, 1980; revised October 29, 1980. This work was sponsored by the Department of the Army. The views and conclusions in this document are those of the contractor and should not be interpreted as necessarily representing the official policies, either expressed or implied, of the United States Government.

The author is with the Massachusetts Institute of Technology, Lincoln Laboratory, Lexington, MA 02173.
TABLE I

MEASURED IMPEDANCES OF GaAs SchotTKY DIODES AT $10 \mathrm{GHz}$

\begin{tabular}{cc}
\hline \hline Diode Current (mA) & Impedance (Ohms) \\
\hline 0.0 & $6 .-\mathrm{j} 51$. \\
0.5 & $17 .-\mathrm{j} 45$. \\
1.0 & $29 .-\mathrm{j} 34$. \\
2.0 & $35 .-\mathrm{j} 11$. \\
\hline
\end{tabular}

states of the terminating impedances. The condition is given which determines the reflection coefficients that are available from any pair of impedance states. This condition is shown to coincide with the definition of the $Q^{2}$ of the terminating impedance pair introduced by Kurokawa and Schlosser.

The switching performance of the reflection modulator depends inherently upon the impedance levels presented at its reflecting terminations. The impedance characteristics of semiconductor devices available for use are significant for this application. The silicon $\mathrm{p}-\mathrm{i}-\mathrm{n}$ diode is frequently employed in switching circuits because its impedance states approach nearly ideal metal switch contact behavior. Interest is presently growing in the adoption of GaAs semiconductor devices in microwave circuits. It is, therefore, relevant to determine whether impedance properties typical of GaAs devices are useful in the reflection modulator circuits being considered. Typical measured values of GaAs Schottky beam-leaded diode impedances at $10 \mathrm{GHz}$ are shown in Table $\mathbf{I}$. 


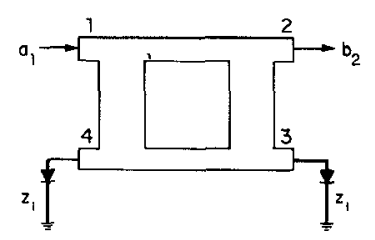

(a)

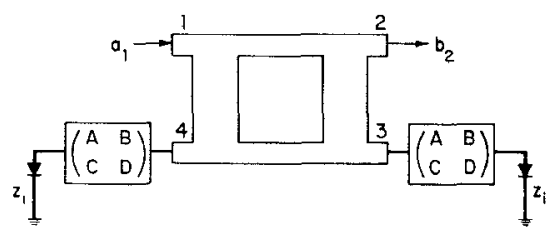

(b)

Fig. 1. Hybrid-coupled impedances. (a) Direct terminations. (b) Impedance-transformed terminations.

Because of its high impedance in all bias states, the Schottky diode is not applicable as a simple series or shunt switch analogous to the silicon p-i-n diode. Useful reflection modulators can be constructed using nonideal semiconductors, however, by introducing impedance transformation ahead of the semiconductor termination (Fig. 1). It will be assumed in the following that the terminating impedances of the reflection modulator are operated between a selected pair of impedance states designated $Z_{1}$ and $Z_{2}$ which can be reached at will by the application of suitable biases. In the case of the digital switching (amplitude) modulator, ideal absorption-reflection (A/R) switch characteristics are wanted. The ideal $A / R$ switch is a two-port junction having scattering matrices in the oFF and oN states, respectively, of the form

$$
\text { OFF : }
$$

$$
S=\left(\begin{array}{ll}
0 & 0 \\
0 & 0
\end{array}\right) \quad S=\left(\begin{array}{ll}
0 & 1 \\
1 & 0
\end{array}\right) .
$$

The semiconductor devices terminating an $A / R$ switch must be capable of dissipating the entire signal power being transferred.

The diode impedance states shown in Table I for bias currents of 2.0 and $0.0 \mathrm{~mA}$ have reflection coefficients of 0.22 and 0.89 , respectively. If used as a reflection modulator, their switching matrices would have the magnitudes

OFF :

$$
S=\left(\begin{array}{cc}
0 . & 0.22 \\
0.22 & 0
\end{array}\right) \quad S=\left(\begin{array}{cc}
0 . & 0.89 \\
0.89 & 0 .
\end{array}\right)
$$

It was suggested in 1965 by Kawakami [9] that the off-state isolation of a switching modulator could be improved by impedance matching the off-state impedance to the system characteristic impedance $Z_{0}$. If the oFF impedance state $Z_{1}$ is transformed to $Z_{0},\left(g_{1}=0\right)$, it can be shown that the magnitude of the reflection coefficient of impedance state $Z_{2}$ transformed by the same impedance transformer is

$$
\left|g_{2}\left(Z_{2}^{\prime}\right)\right|=\left|\frac{Z_{2}-Z_{1}}{Z_{2}+Z_{1}^{*}}\right|
$$

where $Z_{1}^{*}$ is the complex conjugate of $Z_{1}$. This expression (1) remains invariant under transformation of the impedances by any lossless, reciprocal network. Kawakami, therefore, proposed it as a figure of merit for the impedance state pair $Z_{1}, Z_{2}$. Equation (1) predicts a transformed reflection coefficient magnitude $\left|g_{2}\right|$ of 0.86 in the example above. For some applications, an arbitrarily selected reflection coefficient pair may be required. In the following sections it is shown what pairs of reflection coefficients $\left(g_{1}, g_{2}\right)$ may be obtained from a given pair of impedance states $\left(Z_{1}, Z_{2}\right)$ through transformation by a lossless transformer network and how the design parameters of the network may be determined.

\section{IMPEDANCE TRANSFORMER NeTWORK DESIGN}

The derivation of the design parameters for the impedance-transforming network is based on the expression for the transformed reflection coefficient $g_{l}\left(Z_{l}^{\prime}\right)$ appearing at the input to the network when it is terminated by impedance state $Z_{l}(i=1,2)$

$$
g_{l}\left(Z_{\imath}^{\prime}\right)=\frac{Z_{i}^{\prime}-Z_{0}}{Z_{t}^{\prime}+Z_{0}} \quad(i=1,2)
$$

where $Z_{0}$ is the system characteristic impedance, assumed to be real. If the impedance transformer is described by a generalized network $(A, B, C, D)$ matrix, the transformed impedance is given by

$$
Z_{i}^{\prime}=\frac{A Z_{1}+B}{C Z_{i}+D} \quad(i=1,2) .
$$

When (3) is used in (2), the result is

$$
g_{i}=\frac{\left(A-C Z_{0}\right) Z_{i}-\left(D Z_{0}-B\right)}{\left(A+C Z_{0}\right) Z_{i}+\left(D Z_{0}+B\right)} \quad(i=1,2) \text {. }
$$

When the transformation network is lossless, the elements $(A, B, C, D)$ of the network matrix reduce to $(a, j b, j c, d)$ where $a, b, c$, and $d$ are pure-real quantities. For the lossless network, (4) becomes

$$
g_{\imath}=\frac{\left(a-j c Z_{0}\right) Z_{\imath}-\left(d Z_{0}-j b\right)}{\left(a+j c Z_{0}\right) Z_{\imath}+\left(d Z_{0}+j b\right)} \quad(i=1,2)
$$

Defining new quantities $Z_{m}$ and $\eta,(5)$ may be simplified to

$$
g_{l}=\frac{Z_{t}-Z_{m}}{Z_{l}+Z_{m}^{*}} \eta \quad(i=1,2)
$$

where $Z_{m}=\left(d Z_{0}-j b\right) /\left(a-j c Z_{0}\right), Z_{m}^{*}$ is the complex conjugate of $Z_{m}$, and $\eta$ is a phase factor of unit amplitude and angle $\theta=-2 \tan ^{-1}\left(c Z_{0} / a\right)$. In (6), $Z_{m}$ is an impedance parameter equal to the impedance that would be transformed to $Z_{0}$ by the network, for which case the reflection coefficient $g$ vanishes.

When (6) is written for terminations $Z_{1}$ and $Z_{2}$, the two cases may be combined by eliminating the common factor $\eta$, resulting in a solution for $Z_{m}=R_{m}+j X_{m}$ of the form

$$
Z_{m}=-\alpha \pm \sqrt{\alpha^{2}+\beta}
$$


where $\alpha$ and $\beta$ are functions of $Z_{1}, Z_{2}, g_{1}$, and $g_{2}$ (see Appendix). The two-parameter description of the network in terms of $R_{m}$ and $X_{m}$ is employed here instead of a description in terms of the four $A, B, C, D$ parameters of the network. The expressions are thereby simplified and it is a somewhat more convenient task to design a network to match a given impedance $Z_{m}$ than to reproduce a given $(A B C D)$ matrix. This restricts the choice of matching circuits to those which can be described in terms of two parameters. There is a sufficient range of two-parameter circuits to satisfy most matching requirements [8].

\section{UNIQUENESS CONDITION FOR MATCHING NETWORK}

Unique and well-defined solutions for $Z_{m}$ in (7) are not available for all arbitrarily chosen values of $g_{1}$ and $g_{2}$ with given impedance states $Z_{1}$ and $Z_{2}$. The allowable values of $g_{1}$ and $g_{2}$ are restricted by a condition which arises from (5). When (5) is written for cases $i=1$ and $i=2$ and the real and imaginary parts separated, the result is a set of four homogeneous equations in the unknown network parameters $a, b, c$, and $d$. These equations will have a solution only if the determinant of their coefficients vanishes. 'The vanishing of the determinant leads to the relation (see Appendix)

$$
\frac{\left|\sigma_{1}-\sigma_{2}\right|^{2}}{\sigma_{1 r} \sigma_{2 r}}=\frac{\left|Z_{1}-Z_{2}\right|^{2}}{R_{1} R_{2}}
$$

where $\sigma_{1}$ and $\sigma_{2}$ are reflection variables defined by

$$
\sigma_{1}=\frac{g_{1}-1}{g_{1}+1} \quad \sigma_{2}=\frac{g_{2}-1}{g_{2}+1} .
$$

In (8), $\sigma_{1}, \sigma_{2}, g_{1}$, and $g_{2}$ are phasor quantities in general and $\sigma_{1 r}$ and $\sigma_{2 r}$ are the real parts of $\sigma_{1}$ and $\sigma_{2}$, respectively.

When (8) is rewritten in terms of $g_{1}$ and $g_{2}$ it takes the form

$$
\frac{4\left|g_{1}-g_{2}\right|^{2}}{\left(\left|g_{1}\right|^{2}-1\right)\left(\left|g_{2}\right|^{2}-1\right)}=\frac{\left|Z_{1}-Z_{2}\right|^{2}}{R_{1} R_{2}}=Q^{2}
$$

This expression (10) is identical with a relation found by Kurokawa and Schlosser [10], who defined it as the square of the $Q$ of the two-state impedance pair $Z_{1}, Z_{2}$. These authors proposed (10) as the specification of a quality factor for a switching diode which presents impedance states $Z_{1}$ and $Z_{2}$. In the present analysis, $(10)$ is seen also to be a necessary condition for the existence of a lossless network to generate the transformed reflection coefficients $g_{1}$ and $g_{2}$ from $Z_{1}$ and $Z_{2}$. Kurokawa and Schlosser cited the importance of the assumed lossless character of the transformer network in determining (10). The assumption of losslessness is seen here to limit to four the number of

\footnotetext{
'Assigning a zero value to the system determinant reduces the rank of its matrix to 3 . This in principle allows 3 unknowns to be determined in terms of another; e.g., $a / d, b / d, c / d$. Then the condition for a reciprocal, lossless circuit: $a d+b c=1$, allows the determination of the fourth unknown.
}

parameters $(a, b, c, d)$ needed to describe the circuit. Since only four simultaneous equations are available from the separation of the real and imaginary parts of cases $i=1$ and $i=2$ of (5), this enables (5) to yield the system determinant of the equations, providing the existence condition (8) and (10). The assumption of a lossless transformation network is not a serious restriction since in microstrip or waveguide format the network will normally be constructed of sections of microstrip or waveguide transmission line for which the assumed lossless propagation is an acceptable approximation.

The necessary condition (10) implies a specific relationship between $Z_{1}, Z_{2}, g_{1}$, and $g_{2}$. Therefore, it is important to avoid specifying noncommensurate values of $g_{i}$ and $Z_{i}$ (i.e., values which do not satisfy (10)) in the calculation of $Z_{m}$ by means of (7)). A $Z_{m}$ can be calculated by using noncommensurate values of $g_{l}$ and $Z_{l}$ in (7), but a matching network designed from this $Z_{m}$ will produce reflection coefficients $g_{1}$ and $g_{2}$ not in exact agreement with the values initially substituted into (7), when the network is terminated by $Z_{1}$ and $Z_{2}$.

\section{Phase Modulator}

The symbols $Q_{g}^{2}$ and $Q_{Z}^{2}$ may be defined for the left and right sides of $(10)$, respectively

$$
\begin{aligned}
Q_{g}^{2} & =\frac{4\left|g_{1}-g_{2}\right|^{2}}{\left(\left|g_{1}\right|^{2}-1\right)\left(\left|g_{2}\right|^{2}-1\right)} \\
Q_{Z}^{2} & =\frac{\left|Z_{1}-Z_{2}\right|^{2}}{R_{1} R_{2}} .
\end{aligned}
$$

The rectangular coordinates of $g_{1}$ and $g_{2}$ may be written

$$
g_{l}=\left|g_{l}\right| \cos \theta_{t}+j\left|g_{t}\right| \sin \theta_{t} \quad(i=1,2) .
$$

Using these forms in (11) with $Q_{Z}^{2}$ for $Q_{g}^{2}$ leads to the result

$$
\left|g_{1}\right|^{2}-2\left|g_{1}\right|\left|g_{2}\right| \cos \phi+\left|g_{2}\right|^{2}=\frac{Q_{Z}^{2}}{4}\left(1-\left|g_{1}\right|^{2}\right)\left(1-\left|g_{2}\right|^{2}\right)
$$

where $\phi=\left(\theta_{1}-\theta_{2}\right)$ is the phase difference between the two reflection coefficients. In the ideal phase shifter, a change in the phase of the output signal is required with signal amplitude held constant. For the reflection phase shifter this implies

$$
\left|g_{1}\right|=\left|g_{2}\right|=|g| \text {. }
$$

Equation (14) provides a specific relationship between the relative signal amplitude available from a reflection-type phase shifter and the phase shift $\phi$, for a given impedance pair. From (14) and (14a) this relation is

$$
|g|=\left|\frac{m}{2}-\sqrt{1+\frac{m^{2}}{4}}\right|
$$

where $m^{2}=8(1-\cos \phi) / Q_{Z}^{2}$. The minimum transfer loss in decibels of a reflection phase shifter is therefore $20 \log |g|$. A typical dependence of relative signal amplitude on phase 


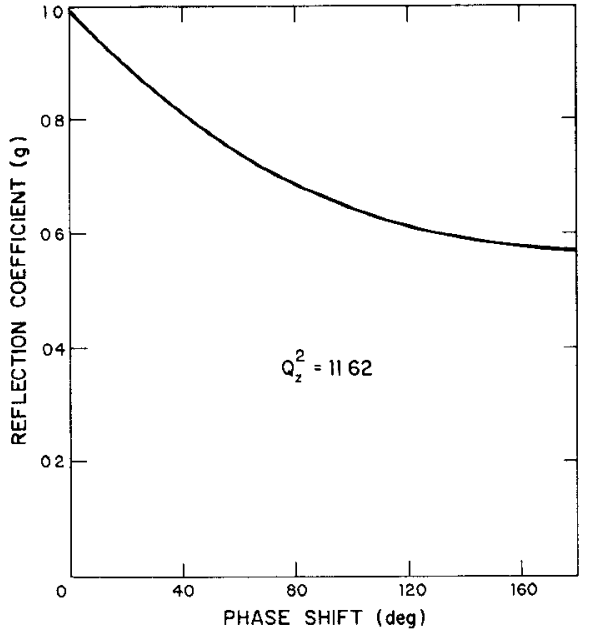

Fig. 2. Transfer factor $g$ versus phase shift for a reflection phase shifter based on an impedance pair having $Q_{Z}^{2}=11.62$.

shift for the reflection-type phase shifter is shown in Fig. 2, which was calculated from (15) with $Q_{Z}^{2}=11.62$, corresponding to the 0.5 and $2.0-\mathrm{mA}$ impedance pair in Table I, with (12).

\section{Amplitude Modulator}

In (14), the absolute phases of $g_{1}$ and $g_{2}$ do not appear, but only their difference $\phi$. From (14), if the relative signal levels $\left|g_{1}\right|$ and $\left|g_{2}\right|$ at the output of an amplitude modulator are specified, the phase shift accompanying the modulation is then given by

$$
\cos \phi=\frac{\left|g_{1}\right|^{2}+\left|g_{2}\right|^{2}-\left(Q_{Z}^{2} / 4\right)\left(1-\left|g_{1}\right|^{2}\right)\left(1-\left|g_{2}\right|^{2}\right)}{2\left|g_{1}\right|\left|g_{2}\right|}
$$

Solutions for a transformation network for the amplitude modulator do not exist for all arbitrarily chosen values of $g_{1}$ and $g_{2}$, but the existence of solutions and the associated phase shift $\phi$ may be found from (16) by trial. Alternatively, if a specific value of $\phi$ is assigned, the attainable $\left|g_{2}\right|$ for a given $\left|g_{1}\right|$ may be found from (14) to be

$$
\left|g_{2}\right|=\left|G \pm \sqrt{F+G^{2}}\right|
$$

where

$$
\begin{aligned}
G & =\frac{\left|g_{1}\right| \cos \phi}{1+p_{1}^{2}} \\
F & =\frac{p_{1}^{2}-\left|g_{1}\right|^{2}}{1+p_{1}^{2}} \\
p_{1}^{2} & =\frac{Q_{Z}^{2}}{4}\left(1-\left|g_{1}\right|^{2}\right) .
\end{aligned}
$$

The assignment of $\phi=0$ is a convenient choice for the amplitude modulator, implying an amplitude transition with no phase shift. A $\phi=0$ always provides a solution for $\left|g_{2}\right|$ in (17).

\section{EXAmples of Modulator Design}

As an example of the versatility of the procedure outlined in the preceding sections, it can be shown that a single pair of impedance states selected from Table I can be used with suitable transformation networks to produce a) an amplitude modulator, b) a $45^{\circ}$ phase shifter, or c) a $90^{\circ}$ phase shifter.

The impedance states from Table I which will be employed are

$$
\begin{aligned}
& Z_{1}=35-j 11 \\
& Z_{2}=6-j 51
\end{aligned}
$$

\section{A. Amplitude Modulator}

The reflection coefficient magnitude $\left(g_{1}\right)$ for the low-level state is arbitrarily assigned a value of 0.05 . It may be assumed that no phase difference exists between $g_{1}$ and $g_{2}$. With no loss of generality, a phase angle of zero may be assigned to both. Therefore, using the $Z_{1}$ and $Z_{2}$ above, $g_{1}=0.05$ (scalar), and $Q_{Z}^{2}=11.62$, (17) may be employed to find: $g_{2}=0.875$. With these values, the solution of (A.14) to (A.19) is: $Z_{m}=(34.33-j 7.59)$. A solution for $Z_{m}$ with negative real part also exists, but this is discarded since it is unrealizable by a passive network. A network is then required which will transform the $Z_{m}$ of $(34.33-j 7.59)$ ohms to the system $Z_{0}$ of $50 \Omega$. This can be done by means of a section of transmission-line transformer of characteristic impedance $39.2 \Omega$ and electrical length $122^{\circ}$. This impedance-transforming circuit is easily realizable in microstrip. The validity of the reflection-coefficient transformer can be verified by testing the $Z_{m}$ value above in Kawakami's condition (1) rewritten for a $Z_{m}$-matching network

$$
\left|g_{l}\left(Z_{l}^{\prime}\right)\right|=\left|\frac{Z_{i}-Z_{m}}{Z_{\imath}+Z_{m}^{*}}\right|
$$

Using the given $Z_{1}$ and $Z_{2}$ values, the transformed coefficients are confirmed to be $g_{1}=0.05, g_{2}=0.875$, or an isolation of $26 \mathrm{~dB}$ and transmission loss of $1.2 \mathrm{~dB}$. This confirmation, as cited in Section III, is the result of having selected $g_{l}, g_{2}, Z_{l}$, and $Z_{2}$ which satisfy (10).

\section{B. $45^{\circ}$ Phase Shifter}

For a $45^{\circ}$ phase shift with $Q_{Z}^{2}=11.62,\left|g_{1}\right|=\left|g_{2}\right|=0.80$, from (15). Therefore, the minimum attenuation for the $45^{\circ}$ phase shifter will be $-1.94 \mathrm{~dB}$. Suitable values of $g_{1}$ and $g_{2}$ for use in (7) are

$$
\begin{aligned}
& g_{1}=0.80 \angle 0^{\circ}=0.80+j 0.0 \\
& g_{2}=0.80 \angle 45^{\circ}=0.566+j 0.566 .
\end{aligned}
$$

Using these data and the chosen $Z_{1}, Z_{2}$ in (7) produces the network parameter: $Z_{m}=(18.15-j 76.10)$. A simple matching network to transform this impedance to $Z_{0}=50 \Omega$ can be constructed of a single open-ended stub of electrical length $70^{\circ}$ connected in shunt with the line at $75.8^{\circ}$ from the termination. 


\section{C. $90^{\circ}$ Phase Shifter}

From (15), a $90^{\circ}$ phase shifter with the $Z_{1}$ and $Z_{2}$ in use may have a reflection amplitude $\left|g_{1}\right|=\left|g_{2}\right|=g=0.668$, or -3.5-dB minimum loss. This data in (7) leads to a network parameter: $Z_{m}=24.48-j 62.46$. This transformation can be realized with a single open stub of electrical length $62.6^{\circ}$ connected at $77^{\circ}$ from the termination.

\section{Conclusion}

A procedure has been given for deriving the design of a generalized two-state reflection-type modulator based on the transformed reflection coefficients of two-state variable impedances. The values of complex reflection coefficient available by transformation from a given two-state impedance pair are shown to be determined by a relation which occurs as a condition for the existence of a solution for the network parameters of the impedance transformers.

\section{APPENDIX}

The general network matrix for a lossless two-port circuit has the form

$$
\left(\begin{array}{ll}
A & B \\
C & D
\end{array}\right)=\left(\begin{array}{cc}
a & j b \\
j c & d
\end{array}\right)
$$

where $a, b, c$, and $d$ are real. The impedance transformation by the two-port is given by

$$
Z_{i}^{\prime}=\frac{a Z_{i}+j b}{j c Z_{i}+d}
$$

When (A.2) is introduced into the expression for reflection coefficient $((2)$ of the text) the result becomes

$$
g_{l}\left(Z_{l}^{\prime}\right)=\frac{\left(a-j c Z_{0}\right) Z_{l}-\left(d Z_{0}-j b\right)}{\left(a+j c Z_{0}\right) Z_{i}+\left(d Z_{0}+j b\right)} .
$$

\section{Necessary Condition for Transformation Network}

Equation (A.3) is equivalent to

$$
\left(g_{i}-1\right) Z_{i} a+j\left(g_{i}-1\right) b+j\left(g_{i}+1\right) Z_{0} Z_{l} c+\left(g_{l}+1\right) Z_{0} d=0 \text {. }
$$

Introducing the reflection variable $\sigma$

$$
\sigma_{t}=\frac{g_{\imath}-1}{g_{\imath}+1}
$$

Equation (A.4) becomes

$$
\sigma_{\imath} Z_{l} a+j \sigma_{i} b+j Z_{0} Z_{t} c+Z_{0} d=0 .
$$

The complex form of $\sigma_{i}$ and $Z_{i}$ may be written

$$
\begin{aligned}
& \sigma_{\imath}=\sigma_{i}^{\prime}+j \sigma_{i}^{\prime \prime} \\
& Z_{\imath}=R_{\imath}+j X_{\imath}
\end{aligned}
$$

when these forms are introduced into (A.6) and the real and imaginary parts separated, the result is:

$$
\begin{gathered}
\text { Real: }\left(\sigma_{t}^{\prime} R_{l}-\sigma_{t}^{\prime \prime} X_{t}\right) a-\sigma_{t}^{\prime \prime} b-Z_{0} X_{l} c+Z_{0} d=0 \\
\text { Imag: }\left(\sigma_{l}^{\prime \prime} R_{l}+\sigma_{l}^{\prime} X_{l}\right) a+\sigma_{l}^{\prime} b-Z_{0} R_{l} c=0 .
\end{gathered}
$$

Writing (A.7) and (A.8) for cases $i=1$ and $i=2$ yields a set of four homogeneous equations in the network con- stants $a, b, c, d$. These equations will have a unique solution only if the determinant of their coefficients vanishes. This determinant is

$$
\left|\begin{array}{cccc}
\left(\sigma_{1}^{\prime} R_{1}-\sigma_{1}^{\prime \prime} X_{1}\right) & -\sigma_{1}^{\prime \prime} & -Z_{0} X_{1} & Z_{0} \\
\left(\sigma_{2}^{\prime} R_{2}-\sigma_{2}^{\prime \prime} X_{2}\right) & -\sigma_{2}^{\prime \prime} & -Z_{0} X_{2} & Z_{0} \\
\left(\sigma_{1}^{\prime \prime} R_{1}+\sigma_{1}^{\prime} X_{1}\right) & \sigma_{1}^{\prime} & Z_{0} R_{1} & 0 \\
\left(\sigma_{2}^{\prime \prime} R_{2}+\sigma_{2}^{\prime} X_{2}\right) & \sigma_{2}^{\prime} & Z_{0} R_{2} & 0
\end{array}\right|=0 .
$$

Expanding (A.9) produces the result

$$
\frac{\left(X_{1}-X_{2}\right)^{2}+\left(R_{1}-R_{2}\right)^{2}}{R_{1} R_{2}}=\frac{\left(\sigma_{1}^{\prime}-\sigma_{2}^{\prime}\right)^{2}+\left(\sigma_{1}^{\prime \prime}-\sigma_{2}^{\prime \prime}\right)^{2}}{\sigma_{1}^{\prime} \sigma_{2}^{\prime}} .
$$

This equation may be written more compactly in the form

$$
\frac{\left|Z_{1}-Z_{2}\right|^{2}}{R_{1} R_{2}}=\frac{\left|\sigma_{1}-\sigma_{2}\right|^{2}}{\sigma_{1}^{\prime} \sigma_{2}^{\prime}}
$$

Equation (A.10) is a necessary condition for the existence of a solution for the network constants of an impedance transformer which produces complex reflection coefficients $g_{1}$ and $g_{2}$ when terminated with impedances $Z_{1}$ and $Z_{2}$.

\section{Solution for Network Parameter $Z_{m}$}

Equation (A.3) may be written

$$
g_{i}\left(Z_{i}^{\prime}\right)=\frac{Z_{i}-Z_{m}}{Z_{l}+Z_{m}^{*}} \frac{\left(a-j c Z_{0}\right)}{\left(a+j c Z_{0}\right)}=\frac{Z_{l}-Z_{m}}{Z_{l}+Z_{m}^{*}} \eta
$$

where $Z_{m}=\left(d Z_{0}-j b\right) /\left(a-j c Z_{0}\right)$ and $\eta$ is a phase factor of unit amplitude: $\eta=\exp \left(2 j \tan ^{-1}\left(-c Z_{0} / a\right)\right)$.

The versions of (A.11) for cases $i=1$ and $i=2$ may be combined

$$
\frac{1}{\eta}=\frac{1}{g_{1}} \frac{Z_{1}-Z_{m}}{Z_{1}+Z_{m}^{*}}=\frac{1}{g_{2}} \frac{Z_{2}-Z_{m}}{Z_{2}+Z_{m}^{*}} .
$$

The solution for $Z_{m}^{*}$ is

$$
Z_{m}^{*}=\frac{M Z_{m}+P}{Z_{m}+N}
$$

where

$$
\begin{aligned}
& M=-\frac{\left(g_{1} Z_{1}-g_{2} Z_{2}\right)}{\left(g_{1}-g_{2}\right)} \\
& N=-\frac{\left(g_{1} Z_{2}-g_{2} Z_{1}\right)}{\left(g_{1}-g_{2}\right)} \\
& P=Z_{1} Z_{2} .
\end{aligned}
$$

Equation (A.13) may be solved for $Z_{m}$ by taking its complex conjugate and substituting back into itself. This leads to the solution

$$
Z_{m}=-\alpha \pm \sqrt{\beta+\alpha^{2}}
$$

where

$$
\begin{aligned}
& \alpha=\frac{1}{2} \frac{P-P^{*}+N N^{*}-M M^{*}}{M+N^{*}} . \\
& \beta=\frac{M^{*} P+N P^{*}}{M+N^{*}} .
\end{aligned}
$$




\section{ACKNOWLEDGMENT}

Acknowledgment is owed to Dr. D. H. Steinbrecher for introduction to the concept of the use of impedance transformation for the predetermination of reflection characteristics in switching. M. L. Smith is thanked for discussions of the analytical aspects of the derivation.

\section{REFERENCES}

[1] B. E. Dobratz, N. J. Ho, G. H. Lee, and H. T. Suyematsu, "Microwave analog and digital signal processors," Proc. IEEE Int. Solld State Circuits Conf., pp. 76-77, 1978.

[2] J. M. Robinson and A. Husain, "Design of direct phase modulators for high speed digital radio systems using MIC techniques," in Proc. IEEE MTT-S Int. Symp. Dig., pp. 220-223, 1977.

[3] J. F. White, "Diode phase shifters for array antennas," IEEE Trans. Microwave Theory Tech., vol. MTT-22, pp. 658-674, June 1974.

[4] B. Glance, "A fast low-loss microstrip PIN phase shifter," IEEE
Trans. Microwave Theory Tech., vol. MTT-27, pp. 14-16, Jan. 1979.

[5] H. Junghaus, "A Ku-band hybrid-coupled 4-phase modulator in MIC technology," in Trans. European Microwave Conf. (Hamburg, Germany), pp. 133-137, 1975.

[6] T. Yahara, Y. Kadomaki, H. Hoshika, and K. Shirahata, "Broadband $180^{\circ}$ phase shift section in X-band," IEEE Trans. Microwave Theory Tech., vol. MTT-23, pp. 307-309, Mar. 1975.

[7] K. Hirai and S. Kamihashi, "Practical design of C-band MIC PIN phase shifters," in Proc. IEEE MTT-S Int. Symp. Dig., pp. 229-231, 1979.

[8] H. Atwater, "Reflection coefficient transformation for phase-shift circuits," IEEE Trans. Microwave Theory Tech., vol. MTT-28, pp. $563-568$, June 1980 .

[9] S. Kawakami, "Figure of merit associated with variable-parameter one-port for R.F. switching and modulation," IEEE Trans. Circuit Theory, vol. CT-12, pp. 321-328, Sept. 1965.

[10] K. Kurokawa and W. O. Schlosser, "Quality factor of switching diodes for digital modulation," IEEE Trans. Microwave Theory. Tech., vol. MTT-18, pp. 180-181, Jan. 1970.

\title{
Analysis of Open-Type Dielectric Waveguides by the Finite-Element Iterative Method
}

\author{
MASATOSHI IKEUCHI, HIDEO SAWAMI, AND HIROSHI NIKI
}

\begin{abstract}
Dispersion characteristics for open-type dielectric waveguide structures operated at millimeter-and submillimeter-wave frequencies are calculated by a finite-element iterative procedure with a given criterion on the maximum field strength at the virtual boundary. Numerical results for a rectangular dielectric image guide are presented and compared with results from other methods. The strip dielectric guide and the insulated image guide with finite- or infinite-width substrates are also analyzed.
\end{abstract}

\section{INTRODUCTION}

V ARIOUS dielectric waveguide structures operated at millimeter-and submillimeter-wave frequencies have been recently developed [1]-[3]. In the structure design, it becomes important to calculate the dispersion characteristics, the field distributions, and other quantities. However, rigorous solutions have not been known except for specific structures [4]. Many approximate and numerical methods [1]-[10] for analyzing the various structures (see Fig. 1.) have been presented in the past decade. Among them, the effective dielectric constant method [1], [2], the transverse resonance method [5], [6], and other methods [3], [7] which cannot provide complete information on the field distributions, and it has been recently suggested [8] that [1], [2] are

Manuscript received July 28, 1980; revised October 29, 1980.

The authors are with the Department of Applied Mathematics, Okayama University of Science, Okayama. 700 Japan.

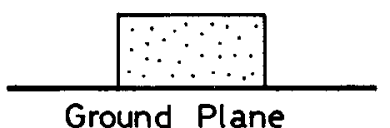

(a)

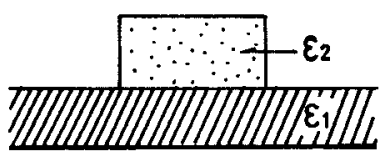

(b)

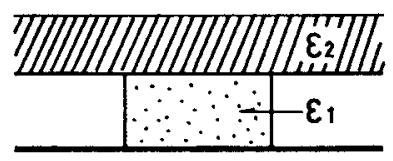

(c)

Fig. 1. Dielectric wavegude structures with conducting ground plane. (a) Dielectric image guide. (b) Strip dielectric guide for $\epsilon_{1}>\epsilon_{2}$, insulated image guide for $\epsilon_{1}<\epsilon_{2}$, single-material guide for $\epsilon_{1}=\epsilon_{2}$. (c) Inverted strip dielectric guide for $\epsilon_{1} \leqslant \epsilon_{2}$

the single-mode approximations. The field-matching method [9] and the method employing the telegraphist's equation [10] are efficient where metallic walls are assumed. The influence of the metallic wall on the inherent 